\title{
Multiphoton Microscopy of Ex Vivo Corneas after Collagen Cross-Linking
}

\author{
Juan M. Bueno, ${ }^{1}$ Emilio J. Gualda,${ }^{1}$ Anastasia Giakoumaki, ${ }^{1}$ Pablo Pérez-Merino, ${ }^{2}$ \\ Susana Marcos, ${ }^{2}$ and Pablo Artal ${ }^{1}$
}

Purpose. To investigate changes in the morphology of the corneal stroma after collagen cross-linking (CXL) treatment in bovine and porcine eyes using a nonlinear microscope providing both two-photon excitation fluorescence (TPEF) and second harmonic generation (SHG) corneal images.

Methods. Freshly enucleated eyes were imaged using a tomographic nonlinear imaging method that was highly suitable to track temporal changes in corneal structures. CXL (riboflavin instillation plus UV irradiation) was applied on the enucleated eyes using similar protocols as in the clinic. A set of eyes without treatment were measured to be used as control.

Results. In control corneas, SHG images showed a regular distribution of lamellae across the stroma that appeared stable for at least 6 hours postmortem. CXL changed the collagen distribution pattern showing some abnormal structures. TPEF revealed a large reduction in corneal thickness in CXL corneas immediately after treatment. The changes in the distribution of collagen bundles appeared also in corneas treated with riboflavin only, but not followed by UV irradiation. SHG tomography also revealed a partial recovery of the corneal thickness with time.

Conclusions. Nonlinear microscopy (in both tomographic and regular XY imaging configurations) was used to study spatial and temporal changes in the cornea during and after CXL on intact ocular globes. SHG imaging showed changes in the morphology of anterior corneal stroma after CXL. Regular collagen patterns turned into random distributed structures with thicker bundles at some localized areas. This might be a consequence of the corneal thickness decrease as a result of riboflavin-dextran instillation. (Invest Ophthalmol Vis Sci. 2011;52:5325-5331) DOI:10.1167/iovs.11-7184

From the ${ }^{1}$ Laboratorio de Óptica, Centro de Investigación en Óptica y Nanofísica, Universidad de Murcia, Campus de Espinardo (Ed. 34), Murcia, Spain; and the ${ }^{2}$ Instituto de Óptica "Daza de Valdés," Consejo Superior de Investigaciones, Científicas, Madrid, Spain.

Presented in part at the annual meeting of the Association for Research in Vision and Ophthalmology, Fort Lauderdale, Florida, May 2010.

Supported by the "Ministerio de Ciencia e Innovación," Spain (Grants FIS2008-02065, FIS2009-06234-E, FIS2010-14926 and Consolider SAUUL CSD2007-00033), "Fundación Séneca," Murcia, Spain (Grant 04524/GERM/06), and EUROHORCS-ESF (Grant EURYI-05102-ES).

Submitted for publication January 7, 2011; revised March 11, 2011; accepted March 26, 2011

Disclosure: J. Bueno, None; E. Gualda, None; A. Giakoumaki, None; P. Perez-Merino, None; S. Marcos, None; P. Artal, None.

Corresponding author: Juan M. Bueno, Universidad de Murcia, Laboratorio de Óptica, Campus de Espinardo (Ed. 34), 30100 Murcia, Spain; bueno@um.es.
K eratoconus is a noninflammatory, progressive thinning disease of the cornea that is characterized by the development of a protrusion with an apex often located centrally or in an inferior eccentric position. ${ }^{1}$ Keratoconic corneas show alterations in the anterior corneal lamellar organization, and in particular reduced lamellar interweaving compared to normal corneas. $^{2,3}$ The observed abnormalities in collagen distribution and orientation have led researchers to propose mechanisms of keratoconus progression that involve interfibrillar or interlamellar slippage, causing a redistribution of tissue within the cornea. ${ }^{4}$ Biomechanical properties are affected and the corneal shape becomes distorted, severely degrading retinal image quality. ${ }^{5}$

Contact lenses and spectacles are the usual treatments in the disease's early stages. In more advanced stages, the implantation of rigid PMMA rings in the stroma ${ }^{6}$ or penetrating keratoplasty (PKP) are required. ${ }^{7}$ However, during the last years, the so-called collagen cross-linking (CXL) technique has been used successfully to stabilize keratoconus progression and prevent ectasia. ${ }^{8}$ This surgical treatment is a minimally invasive method based on the application of riboflavin-dextran (used as a photosensitizer) after corneal epithelium removal, followed by UVA irradiation. UVA irradiation has been reported to cause chemical mechanisms of cross-linking by forming intra- and interfibrillar covalent bonds between collagen fibrils by photosensitized oxidation. ${ }^{9}$ This treatment increases corneal stiffness, ${ }^{10,11}$ slowing down the keratoconus progression, and the effects have been proven "long-term."12

Since CXL treatment has become more common, their effects in terms of biomechanical changes, ${ }^{11-13}$ keratocyte apoptosis, ${ }^{14}$ safety limits of UVA radiation, riboflavin dosage parameters and penetration depth, ${ }^{15,16}$ and the influence on corneal laser sugery ${ }^{17}$ have been studied. Most previous studies addressed the changes in the biomechanical properties of the cornea, primarily through corneal strip extensiometry methods ${ }^{18}$ and, to a lesser extent, whole eye inflation methods. ${ }^{13}$ Bottós et al. ${ }^{19}$ visualized the effects of CXL treatment using immunofluorescence confocal imaging. Wollensak et al. ${ }^{20}$ measured a significant increase in collagen fibril diameter on ultrathin sections of rabbit corneas, particularly in the anterior stroma, after CXL.

Multiphoton microscopy techniques, such as two-photon excitation fluorescence (TPEF) and second harmonic generation (SHG), are powerful minimally invasive tools for corneal imaging. ${ }^{21-24}$ The natural structure of the corneal collagen, with a lack of inversion symmetry center, produces a strong SHG signal. Since Yeh et al. ${ }^{22}$ first reported SHG imaging in rabbit corneas in 2002, different authors have explored the distribution of collagen in normal healthy corneas, including humans. ${ }^{24-28}$ Alteration in the arrangement of stromal collagen bundles in pathologic human corneas, such as keratoconus, bullous keratopathy or stromal edema, has also been studied. ${ }^{27,29,30}$ Moreover, the three-dimensional (3D) structure of the corneal collagen has also been investigated. ${ }^{23} \mathrm{~A}$ recent 
study has reported a rapid (30-minute) distribution of riboflavin throughout the corneal stroma and that CXL reduces the amount of refractive change after myopic LASIK. ${ }^{31}$

However, to our knowledge, there is a lack of studies on the morphologic changes induced in the corneal stroma by CXL treatment, neither using radiographic diffraction nor nonlinear imaging. In particular, nonlinear imaging techniques are nondestructive and allow imaging of corneal structures in the eye in situ, and they are therefore highly appropriate to investigate the effects of CXL on the corneal collagen structure. The aim of this work is to use backscattered nonlinear (SHG and TPEF) microscopy in porcine and bovine corneas to study the stroma organization after CXL treatment. The temporal evolution of the corneal thickness and the 3D arrangement of the collagen structure will be presented.

\section{Materials AND Methods}

\section{Multiphoton Microscope}

A research prototype nonlinear microscope equipped with adaptive optics was used to record the nonlinear signals from the cornea. A detailed description of the instrument can be found elsewhere. ${ }^{32,33}$ The setup used a mode-locked Ti:Sapphire laser (Mira900f; Coherent, St. Clara, CA) as illumination and a photomultiplier tube (R7205-01; Hamamatsu, Shizouka, Japan) as detection unit. The excitation wavelength was set at $760 \mathrm{~nm}$. The instrument was built on a modified inverted microscope (Nikon TE2000-U) using a $20 \times$ objective (Nikon ELWD; numerical aperture $[\mathrm{NA}]=0.5$ ). The SHG signal was collected in the backward direction via the same objective. A dichroic mirror separated the excitation light from the generated nonlinear signal coming back from the sample. The XY scanning of the sample was performed using two nonresonant galvanometric mirrors (VM1000; GSI, Billerica, MA). A DC motor (PI C-136; Karlsruhe, Germany) coupled to the objective position control allowed optical sectioning across the entire cornea along the Z-direction. Two different filters (long-pass and narrow-band) placed in the detection channel allowed for the independent acquisition of either TPEF (FGL435; Thorlabs Inc., Newton, NJ) or SHG (FB380 - 10; Thorlabs Inc.) signals from the specimens under analysis. The system also includes an adaptive optics module ${ }^{34}$ composed of a wavefront sensor and a deformable mirror.

Intact ocular globes were used for this experiment. CXL-treated corneas and those with only riboflavin-dextran-instilled (epithelium removed and no UV irradiation) were compared to normal corneas used as control. In these control eyes, the epithelium was also previously removed. Labview (Austin, TX) and Matlab (The MathWorks, Inc., Natick, MA) custom-developed software were used for image acquisition, instrument control, and postprocessing. 3D images were reconstructed using ImageJ free software.

\section{Eyes}

Fresh bovine ( 4 specimens) and porcine (12 specimens) ocular globes were obtained from a local abattoir. All eyes were excised from 6-month old animals. The eyes were enucleated just after death and transported to the laboratory within less than an hour postmortem. During transportation, the samples were kept in a refrigerated chamber at $4^{\circ} \mathrm{C}$ with cotton soaked in physiologic saline medium (sodium chloride $0.9 \%$ ). Samples were not stained or fixed, and the epithelium was always removed before multiphoton imaging. All corneas appeared clear during the entire experiment.

\section{Treatment}

The CXL treatment was performed in accordance with the standard clinical procedures. ${ }^{8}$ The corneal epithelium was firstly removed with a scalpel to allow the photosensitizer solution to diffuse into the stroma and facilitate UV light exposure. Then a solution containing $0.125 \%$ riboflavin (vitamin $\mathrm{B}_{2}$ ) and $20 \%$ dextran was instilled every 5 minutes for 30 minutes. Finally the corneas were irradiated with UVA light for 30 minutes using a UV-X radiation system (IROC, Zurich, Switzerland). This provides a UV irradiance of $2.038 \mathrm{~mW} / \mathrm{cm}^{2}$ over an area of $12 \mathrm{~mm}$ in diameter. During UV irradiation, riboflavin was also instilled every 5 minutes.

\section{Imaging Protocol}

Two different imaging modes were used to record both TPEF and SHG signals: a "regular" $\mathrm{XY}$ imaging along the $\mathrm{Z}$ axis and a XZ tomographic imaging (similar to B scanning). These two imaging modes require different temporal acquisition patterns. The acquisition time of an $\mathrm{XY}$ image $(500 \times 500$ pixels $)$ is approximately 4 seconds, and therefore a through-focus acquisition of $750 \mu \mathrm{m}$ of corneal thickness (at $10 \mu \mathrm{m}$ Z-step and 3 frames/plane) takes approximately 15 minutes. However, when using the tomographic method, the total acquisition time is approximately 2 minutes for a recording of the same thickness (with 1 $\mu \mathrm{m}$ lateral separation). Whereas the former allows both the analysis of structural changes across an XY plane for a particular depth position and the reconstruction of $3 \mathrm{D}$ volume renderings of the cornea, the latter permits a faster visualization of variations in corneal thickness and the analysis of transversal changes in the corneal morphology after treatment or manipulation. The entire CXL treatment together with the acquisition of series of $\mathrm{XY}$ images across the whole cornea usually took between 1.25 and 2 hours. To verify if changes in the stroma were exclusively caused by CXL treatment, a control eye was imaged over a period of 4 hours.

Because the microscope worked in a backscattered mode, the ocular globes were placed in a bottom-glass dish filled with saline solution (to ensure liability and humidity) in an upside-down position (i.e., the corneal Bowman's layer lying in contact with the cover glass). However, for the sake of clarity, all $\mathrm{XZ}$ tomographic images in the

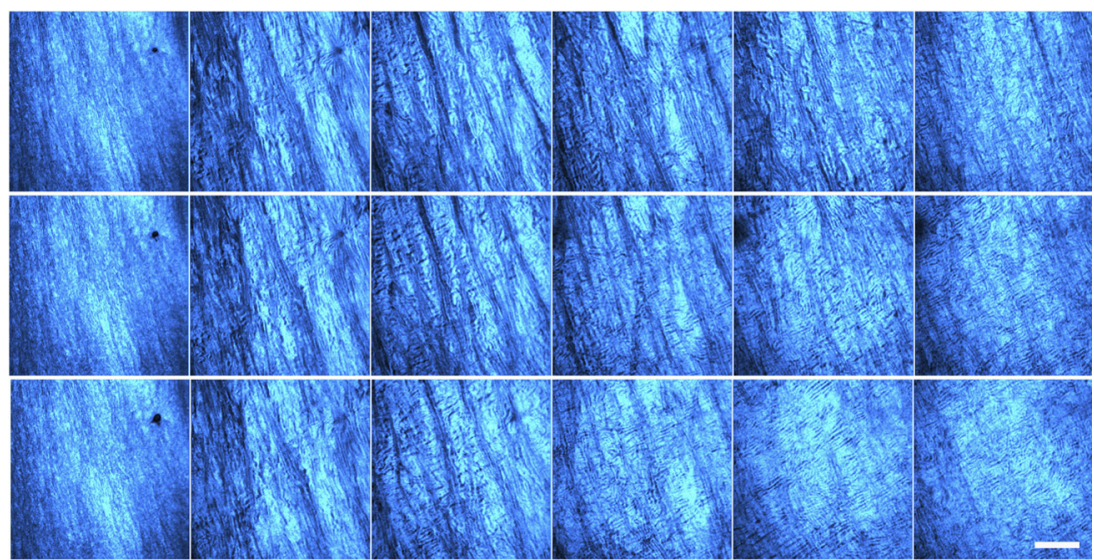

Figure 1. SHG images of the corneal stroma of a untreated porcine eye at different depths (spaced 40 $\mu \mathrm{m}$ and starting $10 \mu \mathrm{m}$ below the Bowman's layer) acquired at 0,2 , and 3 hours (top, middle, and bottom rows, respectively). Scale bar: $100 \mu \mathrm{m}$. 
Figure 2. SHG images of the corneal stroma in an untreated bovine cornea (upper panel) and in a bovine cornea immediately after CXL treatment (lower panels). Depth positions are (from left to right) 15,75 , $135,195,255$, and $315 \mu \mathrm{m}$ from the Bowman's layer. Scale bar: $100 \mu \mathrm{m}$.

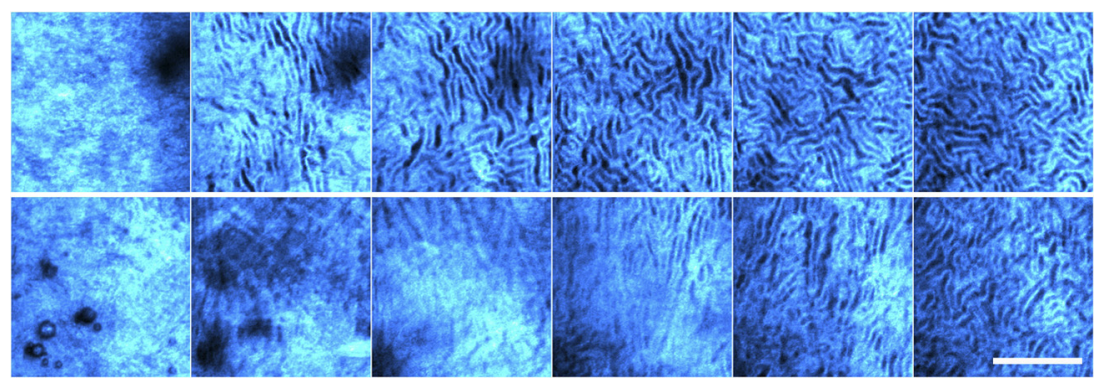

Results section will be represented in such a way that the anterior stroma corresponds to the upper part of the image and the lower part to the endothelium. For all specimens, the imaged areas corresponded to the central cornea. Throughout this work, the $0-\mu \mathrm{m}$ position for corneal depth corresponds to the first Z-position where SHG signal is detected; that location approximately corresponds to the Bowman's layer.

\section{Results}

Figure 1 shows three series of SHG images of corneal stroma at different depths captured at 0, 2, and 3 hours in an untreated porcine eye (with scraped epithelium). The arrangement of the collagen fibers did not change with time, except for some minor modifications at deeper imaged areas after 3 hours. For all images, the stroma arrangement was clearly outlined. In particular, for the deepest positions the lamellae were densely packed and showed an interwoven distribution. The width of the bundles was around $5 \mu \mathrm{m}$.

Figure 2 compares the SHG images of a bovine cornea before (top row) and immediately after CXL treatment (bottom row). The images correspond to corneal depth positions 60 $\mu \mathrm{m}$ apart starting at the Bowman's layer. CXL caused noticeable changes in the arrangement of the collagen fibers, particularly in the anterior stroma. The collagen bundles appear longer, less delineated, and less interwoven after CXL compared to the intact tissue. At $315 \mu \mathrm{m}$ of the Bowman's layer, there is no appreciable difference between treated and untreated corneas. Figure 3 shows the corresponding SHG microscopy images untreated and immediately after CXL in porcine corneas, for depth positions $40 \mu \mathrm{m}$ apart. The arrangement of the stromal collagen fibers lying mostly parallel to the corneal surface of untreated corneas is lost with the CXL treatment. After CXL, the map of collagen fibers presents a random distribution which clearly contrasts with the regular orientation in an untreated cornea (Fig. 1 and upper panels in Fig. 3).

Figure 4 presents 3D volume renderings of both porcine and bovine corneas, before and after CXL treatment, for an axial range of 550 and $660 \mu \mathrm{m}$, respectively. For bovine corneas, we used a set of SHG images recorded $10 \mu \mathrm{m}$ apart, and for porcine corneas, $15 \mu \mathrm{m}$ apart. Figures $4 \mathrm{~B}$ and $4 \mathrm{D}$ show a compression of the stromal collagen of the cornea after the CXL treatment. This decrease was one-third of the original thickness for the porcine cornea and around one half for the bovine cornea.

Figure 5 presents nonlinear tomographic images recorded for different experimental conditions in porcine corneas. Figures $5 \mathrm{~A}$ and $5 \mathrm{~B}$ show the tomography images for TPEF and SHG signal, respectively, for an untreated cornea after epithelium removal and immediately before the CXL treatment. Autofluorescence across the stroma, in Figure $5 \mathrm{~A}$, is related to the presence of keratocytes. The signal at the bottom horizontal band of the image originates from the endothelium. Figure $5 \mathrm{~B}$ depicts the corresponding SHG signal recorded for the same cornea. Only signal from the stroma was observed.

Figures 5C and 5D show the nonlinear signals in the same cornea after complete CXL treatment (riboflavin-dextran instillation and UV irradiation). This treated cornea showed a reduction in thickness. The TPEF signal also increased through the entire cornea (compared with Fig. 5A) because of riboflavin fluorescence. Figures $5 \mathrm{~B}$ and $5 \mathrm{C}$ also show that both TPEF and SHG signals originate from the same areas, indicating that riboflavin has correctly penetrated into the corneal stroma. This ensures that the observed corneal thickness reduction is not an artifact from the imaging method but an important effect of the treatment.

Figures $5 \mathrm{E}$ and $5 \mathrm{~F}$ compare the SHG signal (i.e., the structure of the stroma) in another untreated control cornea with the epithelium removed as a function of time. These SHG images were acquired 1 hour apart, which is the time required to complete a CXL treatment. During this time, the specimen was always laying upside-down on the cover glass and being immersed in solution. No significant changes took place during this time, and the corneal morphology is maintained.

After CXL treatment was performed, a progressive recovery of corneal thickness with time occurred. Figure 6 presents a set of tomographic SHG images of the cornea acquired 15 minutes apart. The thickening rate was around $1 \mu \mathrm{m} / \mathrm{second}$.

Figure 7 analyzes the effects of the riboflavin-dextran instillation itself (no UV irradiation) during the time this process lasts (30 minutes) in the CXL treatment (see Methods). Pairs of TPEF-SHG tomography images were recorded every $10 \mathrm{~min}$ utes (i.e., 10, 20, and 30 minutes after the instillation started). Because the sample was moved away from the microscope for the instillation operation, we could not ensure that the three pairs of images correspond exactly to the same central corneal
Figure 3. SHG images of the corneal stroma in an untreated porcine cornea (upper panel) and in a porcine cornea immediately after CXL treatment (lower panels). Depth positions: $10,50,90,130,170$, and 210 $\mu \mathrm{m}$ from the Bowman's layer. Scale bar: $100 \mu \mathrm{m}$.

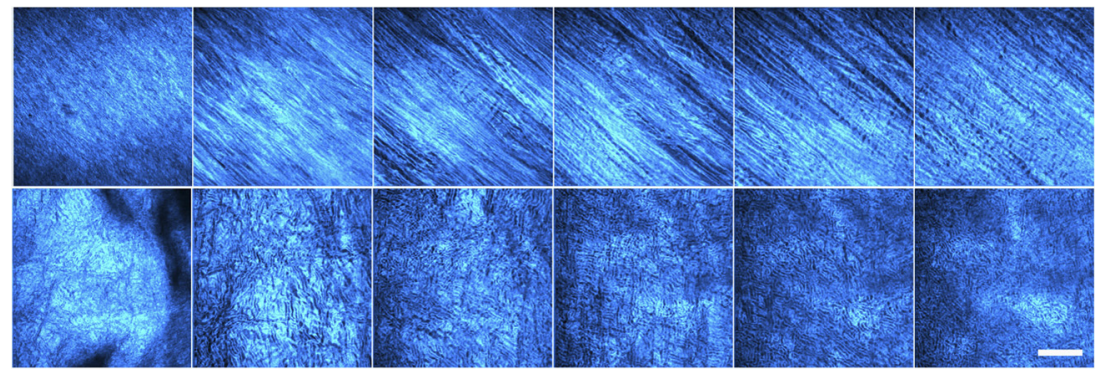



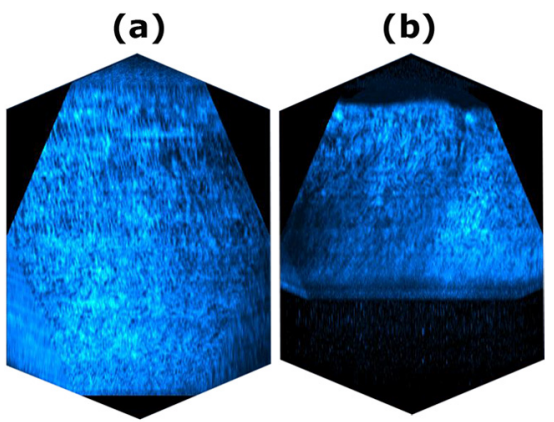

(c)

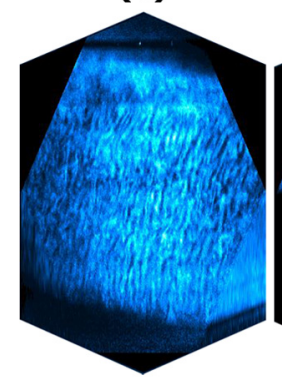

(d)

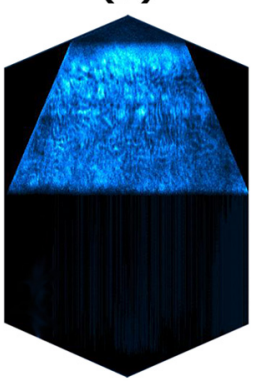

FIGURE 4. 3D reconstruction of SHG signals of porcine (A, B) and bovine $(\mathbf{C}, \mathbf{D})$ corneas, before $(\mathbf{A}, \mathbf{C})$ and after CXL $(\mathbf{B}, \mathbf{D})$. The upper part of the images corresponds to the Bowman's layer. The total imaged thickness was $550 \mu \mathrm{m}$ for (A) and $660 \mu \mathrm{m}$ for (C).

location, although this is not relevant for this particular analysis. Because SHG signal originates from the entire stroma, Figures $7 \mathrm{~B}, 7 \mathrm{D}$, and $7 \mathrm{~F}$ must be taken as a reference for the measurement of corneal thickness as a function of instillation time. Figure 7A (TPEF signal) depicts how the riboflavin the solution has diffused throughout the entire depth of the cornea ("green" and "blue" areas are coincident) after the first 10 minutes of instillation.

A progressive reduction of the corneal thickness with time is observed, which reveals that the dehydration effect of the riboflavin-dextran solution is the main cause for corneal thinning. In particular, Figure 7D (20 minutes of instillation) shows that the SHG signal comes from a more reduced corneal area, indicating that thickness is noticeable reduced compared to Figure $7 \mathrm{~B}$. Accordingly, the companion Figure $7 \mathrm{C}$ depicts that riboflavin covers the entire stroma (which is thinner now). When the instillation finishes (30 minutes), the corneal thinning is even more pronounced (Figs. $7 \mathrm{E}$ and $7 \mathrm{~F}$ ).

The effects of riboflavin-dextran instillation on corneal morphology are illustrated in Figure 8, showing the SHG images corresponding to two particular depth positions (100 and 150 $\mu \mathrm{m})$ before and after riboflavin-dextran instillation in a porcine cornea. The evident differences in the arrangement of the collagen bundles before and after instillation of riboflavindextran, and the similarity of the collagen arrangement after riboflavin-dextran instillation (lower panels of Fig. 8) with those after the entire cross-linking procedure (riboflavin-dextran + UV irradiation; lower panels of Fig. 3) indicates that riboflavin-dextran is not only responsible for the reduction of corneal thickness, but also for the majority of the changes in the corneal stromal collagen arrangement after CXL.

\section{Discussion}

A research adaptive optics nonlinear microscope has been used to visualize the structural changes in the corneal stroma after CXL treatment in bovine and porcine ex vivo eyes. The instrument works in backward configuration and provides both TPEF and SHG signals, which have been reported to be useful for noninvasive corneal imaging. ${ }^{22-31}$ We have shown structural alterations of CXL-treated corneas, which confirms the potential of this imaging modality for the analysis of the effects of this treatment.

In previous studies, the dynamic changes of corneal structures imaged with multiphoton microscopy were not reported. The dynamic changes occurring during the corneal collagen CXL treatment could be important to better understand the time course of the corneal response to the treatment. The use of a new multiphoton imaging modality based on tomographic sectioning has allowed a much faster recording of in-depth images and therefore tracking dynamic changes across the entire thickness of the sample. For this particular application, we were able to record a tomographic image of the entire cornea in approximately 2 minutes.

SHG microscopy has previously been used to detect differences in the organizational pattern of the stromal lamellae in pathologic corneas, but to our knowledge, this has not been used before to explore modifications in the stroma after CXL treatment.

The use of a backscattering microscope configuration allows for imaging of the intact eye. By applying nonlinear microscopy to CXL-treated cornea specimens in intact eyes, it has been shown that the stroma undergoes important morphologic changes. In particular, SHG signal, which targets the collagen structure, reveals changes in the organization and arrangement of the collagen bundles. On the other hand, TPEF provided quantitative, spatially resolved information of the penetration depth of riboflavin. (a)

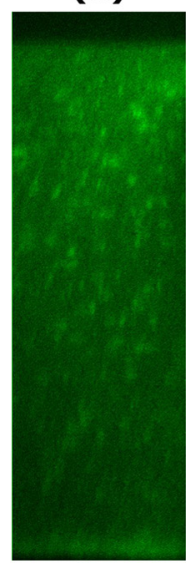

(b)

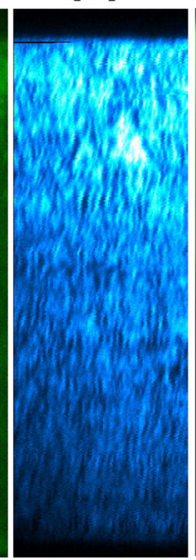

(c)

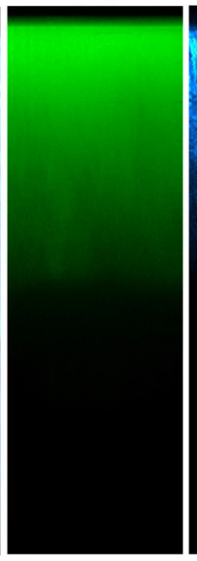

(d)

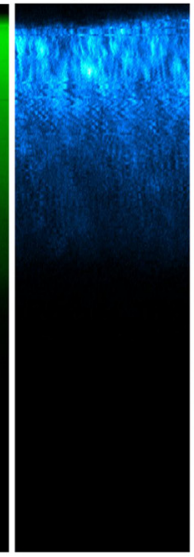

(e)

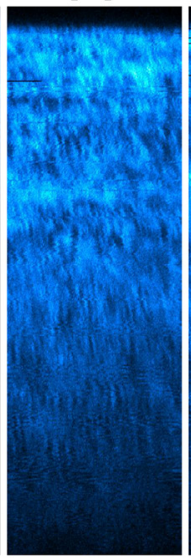

(f)

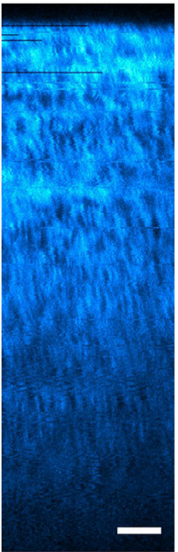

Figure 5. Nonlinear (XZ) tomography images of porcine corneas: TPEF (A) and SHG (B) signals of a control cornea after epithelium removal and before CXL treatment. TPEF (C) and SHG (D) signals from the same cornea as in (A, B) acquired immediately after CXL treatment. SHG signal from another untreated (and epithelium removed) cornea imaged 1 hour apart $(\mathbf{E}, \mathbf{F})$. The top area of the figures corresponds to the Bowman's layer, in contact with the glass-bottom dish. The plotted area is $700 \times$ $215 \mu \mathrm{m}^{2}$. Scale bar: $50 \mu \mathrm{m}$. 
FIGURE 6. SHG tomography images of a porcine cornea after CXL treatment recorded every 15 minutes. The plotted area is $600 \times 230 \mu \mathrm{m}^{2}$. Scale bar: $60 \mu \mathrm{m}$.

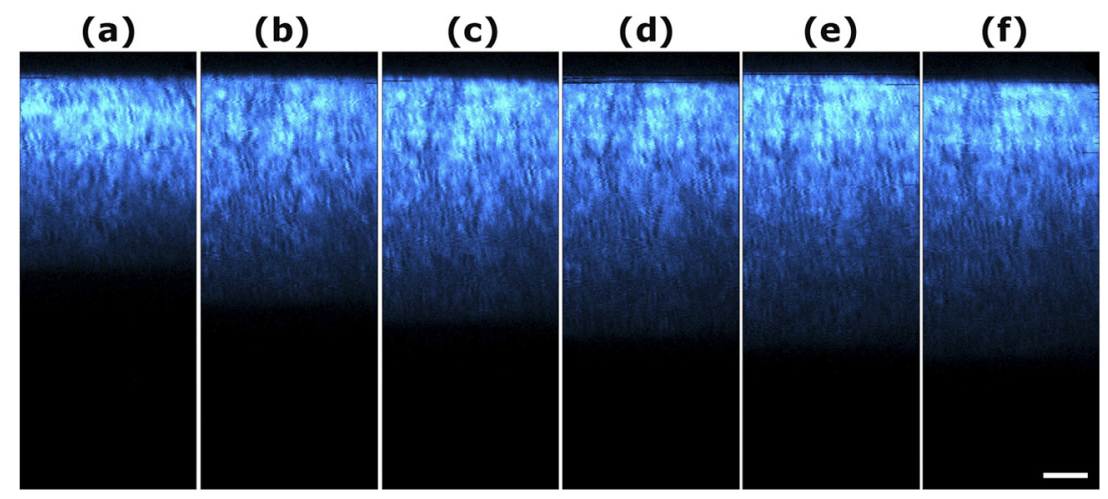

Unlike most previous studies, we were able to image the entire corneal thickness (up to $700 \mu \mathrm{m}$ in control bovine eyes) in the backscattered direction (before and after CXL treatment) with comparatively lower incident energy. Technical improvements, such as the use of a photon-counting unit in the detection pathway together with the wavefront control, ${ }^{32-34}$ allowed us to record good quality images, even at the deepest corneal layers, to visualize the organization of the corneal stroma.

SHG images of bovine and porcine control corneas (Figs. 2 and 3) show the particular packing of stromal lamellae recently reported. ${ }^{28}$ Although this pattern was maintained for several hours in control eyes, it changed in CXL-treated corneas. Randomly distributed, flattened, and abnormal structures of collagen bundles in some localized areas are observed in post-CXL eyes. In particular, it has been shown (Fig. 8) that morphologic changes in the corneal stroma are a result of the riboflavindextran itself, probably caused by dehydration produced by dextran $20 \%$ solution. In bovine eyes, with a thicker stroma containing thicker collagen bundles, the effects were more evident. These variations were only found at the anterior stroma, and the natural corneal fibrous structure seems to remain intact in the posterior stroma. However, these changes are local and difficult to generalize for the entire transversal corneal section given the particular 3D arrangement of the collagen fibrils and the limited scanned area available (up to $410 \times 410 \mu \mathrm{m}^{2}$ ). The thickening of the collagen bundles after CXL have been reported before by Wollensak et al. ${ }^{20}$ in rabbit eyes using biometric measurements of ultrathin corneal sections. The changes in the packing of the collagen fibers in cross-linked areas of the cornea, as opposed to the arrangement of nontreated areas, was recently reported by Steven et al. ${ }^{35}$ using nonlinear microscopy on rabbit eyes.

Our results also show that a major effect in CXL treatment is the transient decrease of corneal thickness during and immediately after the treatment. Although Scheimpflug imaging measurements have recently reported similar results, ${ }^{13}$ this is the first time that multiphoton microscopy has been used to quantify the effect in two and three spatial dimensions, to observe these spatially resolved changes across the corneal thickness and to track those changes as a function of time.

We have shown that corneal thinning is caused primarily by the riboflavin-dextran solution, which is hyperosmolar and consequently has a dehydrating effect on the stroma. The decrease in corneal thickness by instillation of riboflavin (and not the UVA radiation) immediately after treatment has been reported both in vitro in animal models ${ }^{13}$ and intraoperatively in human patients. ${ }^{36}$ There is wide evidence that the dehydrating properties of the dextran $20 \%$ solution in which the riboflavin is diluted are the cause for corneal thinning (Kling $\mathrm{S}$ et al. IOVS 2010;51:ARVO E-Abstract 4628). To prevent this, hypoosmolar solutions are currently under test. ${ }^{37}$ The impact of other possible causes (such as dehydration by evaporation) is negligible. In fact, previous experiments on de-epithelized corneas (neither riboflavin nor CXL) did not show an increase of corneal thickness during the 40-minute period (if anything, corneas tended to slightly increase corneal thickness because of edematization). ${ }^{13}$

For tomographic imaging, it has been shown that TPEF and SHG signals are complementary. Whereas TPEF signal comes
FIGURE 7. Effects of riboflavin-dextran instillation in a porcine cornea as a function of time. TPEF $(\mathbf{A}, \mathbf{C}, \mathbf{E})$ and SHG (B, D, F) tomographic images. Pairs of images were acquired 10 minutes apart (see details in the text). The plotted area is $700 \times 180$ $\mu \mathrm{m}^{2}$. Scale bar: $50 \mu \mathrm{m}$. (a)

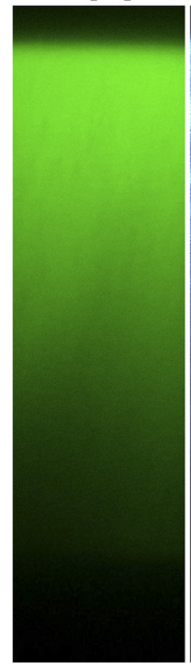

(b)

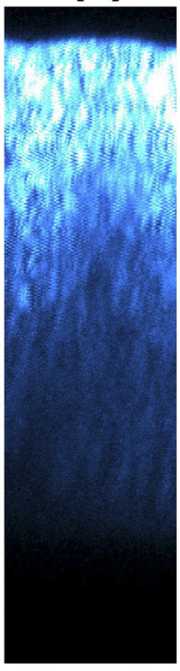

(c)

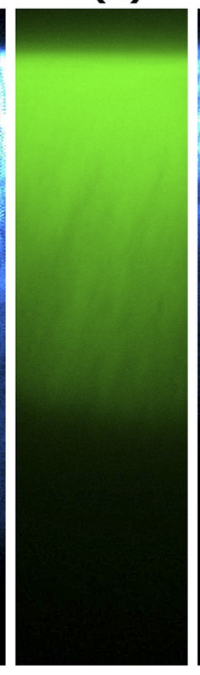

(d)

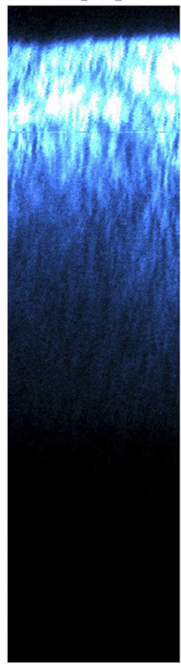

(e)

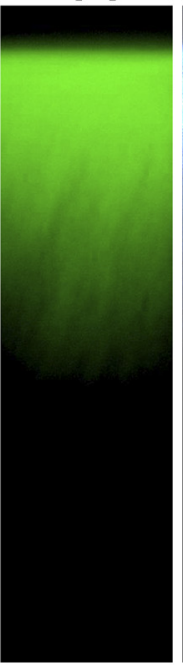

(f)

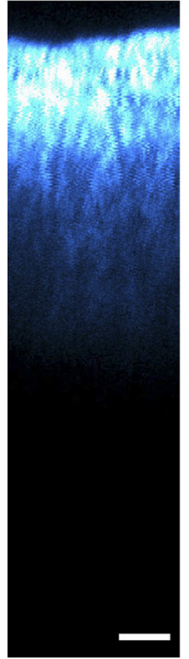




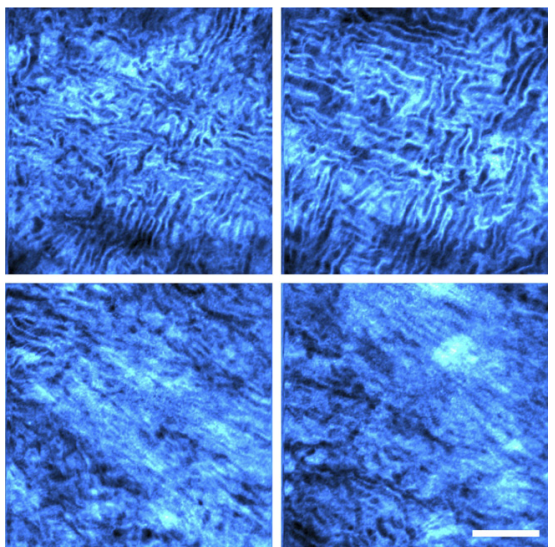

FigURE 8. SHG images of a porcine cornea at depth positions of 100 $\mu \mathrm{m}$ (left panels) and $150 \mu \mathrm{m}$ (right panels) acquired before (upper row) and after riboflavin-dextran instillation (bottom row). Scale bar: $50 \mu \mathrm{m}$.

from endothelial layer and from the keratocytes (the epithelium was removed), SHG is produced in the stroma. After CXL, the thickness reduction is observed in both TPEF and SHG tomography images. Both signals share the same cornea area, which indicates that the riboflavin penetrated into the entire corneal stroma. Moreover, the TPEF signal levels increased through the entire cornea because of the properties of riboflavin fluorescence. This rapid and even distribution of riboflavin through the entire cornea has recently been reported by Kampik et al. ${ }^{31}$ on enucleated porcine eyes.

SHG imaging also revealed a progressive recovery of corneal thickness after CXL treatment. In the ex vivo eyes used here, the increase in corneal thickness was approximately $1 \mu \mathrm{m} /$ second, but the initial thickness was never recovered.

Our results show that riboflavin-dextran solution causes relevant microstructural changes in the collagen. This is consistent with reports from X-ray diffraction ${ }^{38}$ and electron microscopy $^{39}$ on corneas during dehydration that show that in a first stage of dehydration, corneal collagen fibrillar distance decreases as water is only released from the interfibrillar substance, and in a second stage, after dehydration exceeds a critical value, structural transformation of the collagen fibrils occurs with shrinkage and decrease in fibril diameter. Corneal biomechanical properties have been shown to be modulated by corneal hydration. ${ }^{40}$ Furthermore, recent measurements of corneal deformation from inflation experiments show differences in the response of virgin corneas and corneas treated with dextran and riboflavin-dextran, suggesting changes in the interfibrillar distances but not a decrease in fibril diameter in the presence of riboflavin. ${ }^{41}$ CXL has also been shown to increase the stiffening of normal corneas by means of induction of intra- and interfibril bonds, ${ }^{4,9}$ and a similar mechanism may contribute to the observation of ectasia progression in keratoconic corneas.

The morphologic changes in the corneal stroma observed in the present study indicate an increase in the packing of the fibrils. This is consistent with a very recent study using SHG imaging on CXL corneas. ${ }^{35}$ Although the conditions of measurement are different from ours, the study reports changes in the $(\mathrm{XY})$ collagen distribution, from a wavelike to a more homogenous pattern. However, the authors did not address possible changes along the $\mathrm{Z}$ direction.

An open question of the present study is the possible extrapolation of the results on bovine and porcine corneas to human corneas. As recently shown, the collagen arrangement of the cornea strongly depends on the species. ${ }^{33}$ Moreover, corneal thickness and its stiffness (porcine corneas are more elastic than human corneas) are also particular for each species. Although some differences might be expected for human corneas, biomechanical studies of CXL corneas from different species have provided comparable conclusions. Despite differences in the natural organization of collagen fibers in bovine and porcine corneas, we found organizational changes in both species after CXL. The decrease in corneal thickness has been also reported clinically in human patients (Ziebarth NM et al. IOVS 2010;51:ARVO E-Abstract 4998). However, it is unlikely that the intraoperative thinning of the cornea caused by dehydration from dextran is related to redistribution of corneal thickness observed in human patients several months after surgery (in certain corneal locations). These long-term pachymetric changes are likely related to the biomechanical changes leading to symmetrization of the keratoconic corneas after the treatment. $^{42}$

In conclusion, we have shown that multiphoton microscopy is a powerful tool for the study of corneal collagen longitudinal changes after CXL treatment, shedding new light on the mechanisms underlying this treatment. Thanks to the sectioning capabilities of the SHG imaging, it has been possible to reconstruct the $3 \mathrm{D}$ arrangement of the entire corneal stroma and to observe the morphologic changes produced and the evolution of the corneal structure before, during, and after CXL treatment.

\section{References}

1. Colin J, Velou S. Current surgical options for keratoconus. J Cataract Refract Surg. 2003;29:379-386.

2. Radner W, Zehetmayer M, Skorpik C, Mallinger R. Altered organization of collagen in the apex of keratoconus corneas. Ophthalmic Res. 1998;30:327-332.

3. Meek KM, Tuft SJ, Huang Y, et al. Changes in collagen orientation and distribution in keratoconus corneas. Invest Opbthalmol Vis Sci. 2005;46:1948-1956.

4. Hayes S, Boote C, Tuft SJ, Quantock AJ, Meek KM. A study of corneal thickness, shape and collagen organisation in keratoconus using videokeratography and X-ray scattering techniques. Exp Eye Res. 2007;84:423-434.

5. Gefen A, Shalom R, Elad D, Mandel Y. Biomechanical analysis of the keratoconic cornea. J Mech Bebav Biomed Mater. 2009;2: 224-236.

6. Colin J, Cochener B, Savary G, Malet F. Correcting keratoconus with intracorneal rings. J Cataract Refract Surg. 2000;26:11171122.

7. Brierly SC, Izquierdo Jr L, Mannis MJ. Penetrating keratoplasty for keratoconus. Cornea. 2000;19:329-332.

8. Wollensak G, Spoerl E, Seiler T. Riboflavin/ultraviolet-a-induced collagen crosslinking for the treatment of keratoconus. Am J Opbthalmol. 2003;135:620-627.

9. McCall AS, Kraft S, Edelhauser HF, et al. Mechanisms of corneal tissue cross-linking in response to treatment with topical riboflavin and long-wavelength ultraviolet radiation (UVA). Invest Ophthalmol Vis Sci. 2010;51:129-138.

10. Spoerl E, Huhle M, Seiler T. Induction of cross-links in corneal tissue. Exp Eye Res. 1998;66:97-103.

11. Wollensak G, Spoerl E, Seiler T. Stress-strain measurements of human and porcine corneas after riboflavin-ultraviolet-A-induced cross-linking. J Cataract Refract Surg. 2003;29:1780-1785.

12. Wollensak G. Crosslinkining treatment of progressive keratoconus: new hope. Curr Opin Ophthalmol. 2006;17:356-360.

13. Kling S, Remon L, Pérez-Escudero A, Merayo-Lloves J, Marcos S Corneal biomechanical changes after collagen cross-linking from porcine eye inflation experiments. Invest Opbthalmol Vis Sci. 2010;51:3961-3968.

14. Wollensak G, Spoerl E, Wilsch M, Seiler T. Keratocyte apoptosis after corneal collagen cross-linking using riboflavin/UVA treatment. Cornea. 2004;23:43-49. 
15. Spoerl E, Mrochen M, Sliney D, Trokel S, Seiler T. Safety of UVA riboflavin cross-linking of the cornea. Cornea. 2007;26:385-389.

16. Wollensak G, Aurich H, Pham DT, Wirbelbauer C. Hydration behavior of porcine cornea crosslinked with riboflavin and ultraviolet A. J Cataract Refract Surg. 2007;33:516-521.

17. Hafezi F, Kanellopoulos J, Wiltfang R, Seiler T. Corneal collagen crosslinking with riboflavin and ultraviolet $\mathrm{A}$ to treat induced keratectasia after laser in situ keratomileusis. J Cataract Refract Surg. 2007;33:2035-2040.

18. Wollensak G. Corneal collagen crosslinking: new horizons. Expert Rev Ophthalmol. 2010;5:201-215.

19. Bottós KM, Dreyfuss JL, Regatieri CV, et al. Immunofluorescence confocal microscopy of porcine corneas following collagen crosslinking treatment with riboflavin and ultraviolet A.J Refract Surg. 2008;24:S715-9.

20. Wollensak G, Wilsch M, Spoerl E, Seiler T. Collagen fiber diameter in the rabbit cornea after collagen crosslinking by riboflavin/UVA. Cornea. 2004;23:503-507.

21. Piston DW, Masters BR, Webb WW. Three-dimensionally resolved $\mathrm{NAD}(\mathrm{P}) \mathrm{H}$ cellular metabolic redox imaging of the in situ cornea with two-photon excitation laser scanning microscopy. J Microsc. 1995; 178:20-27.

22. Yeh AT, Nassif N, Zoumi A, Tromberg BJ. Selective corneal imaging using combined second-harmonic generation and two-photon excited fluorescence. Opt Lett. 2002;27:2082-2084.

23. Morishige $\mathrm{N}$, Nishida T, Jester J. Second harmonic generation for visualizing 3-dimensional structure of corneal collagen lamellae. Cornea. 2009;28:46-53.

24. Aptel F, Olivier N, Deniset-Besseau A, et al. Multimodal nonlinear imaging of the human cornea. Invest Ophthalmol Vis Sci. 2010; 51:2459-2465.

25. Han M, Giese G, Bille J. Second harmonic generation imaging of collagen fibrils in cornea and sclera. Opt Express. 2005;13:57915797.

26. Teng SW, Tan HY, Ju-Li Peng JL, et al. Multiphoton autofluorescence and second-harmonic generation imaging of the ex vivo porcine eye. Invest Ophthalmol Vis Sci. 2006;47:1216-1224.

27. Morishige N, Petroll WM, Nishida T, Kenney MC, Jester JV. Noninvasive corneal stromal collagen imaging using two-photon-generated second-harmonic signals. J Cataract Refract Surg. 2006;32: 1784-1791.

28. Tan HY, Sun Y, Lo W, et al. Multiphoton fluorescence and second harmonic generation imaging of the structural alterations in keratoconus ex vivo. Invest Ophthalmol Vis Sci. 2006;47:5251-5259.
29. Morishige N, Wahlert AJ, Kenney MC, et al. Second-harmonic imaging microscopy of normal human and keratoconus cornea. Invest Opbthalmol Vis Sci. 2007;48:1087-1094.

30. Morishige N, Yamada N, Teranishi S, Chikama T, Nishida T, Takahara A. Detection of subepithelial fibrosis associated with corneal stromal edema by second harmonic generation imaging microscopy. Invest Opbthalmol Vis Sci. 2009;50:3145-3150.

31. Kampik D, Ralla B, Keller S, Hirschberg M, Friedl P, Geerling G. Influence of corneal collagen crosslinking with riboflavin and ultraviolet-A irradiation on excimer laser surgery. Invest Ophthalmol Vis Sci. 2010;51:3929-3934.

32. Gualda EJ, Bueno JM, Artal P. Wavefront optimized non-linear microscopy of ex-vivo human retinas. J Biom Opt. 2010;15: 026007.

33. Bueno JM, Gualda EJ, Artal P. Analysis of corneal stroma organization with wavefront optimized nonlinear microscopy. Cornea. 2011;30:692-701.

34. Bueno JM, Gualda EJ, Artal P. Adaptive optics multiphoton microscopy to study ex vivo ocular tissues. J Biom Opt. 2010;15:066004.

35. Steven P, Hovakimyan M, Guthoff RF, Hüttmann G, Stachs O. Imaging corneal crosslinking by autofluorescence 2-photon microscopy, second harmonic generation, and fluorescence lifetime measurements. J Cataract Refract Surg. 2010;36:2150-2159.

36. Kymionis GD, Kounis GA, Portaliou DM, et al. Intraoperative pachymetric measurements during corneal collagen cross-linking with riboflavin and ultraviolet A irradiation. Opbthalmol. 2009; 116:2336-2339.

37. Hafezi F, Mrochen M, Iseli HP, Seiler T. Collagen crosslinking with ultraviolet-A and hypoosmolar riboflavin solution in thin corneas. $J$ Cataract Ref Surg. 2009;35:621-624.

38. Meek KM, Fullwood NJ, Cooke PH, et al. Synchrotron x-ray diffraction studies of the cornea with implications for stromal hydration. Biophys J. 1991;60:467-474.

39. Craig AS, Robertson JG, Parry DAD. Preservation of corneal collagen fibril structure using low temperature procedures for electron microscopy. J Ultrastruct Res. 1986;96:172-175.

40. Ahearne M, Yang Y, Then KY, Liu KK. An indentation technique to characterize the mechanical and viscoelastic properties of human and porcine corneas. Ann Biomed Eng. 2007;35:1608-1616.

41. Kling S, Pérez-Merino P, Marcos S. Impact of corneal hydration on corneal biomechanical properties from in vitro inflation tests. $J$ Cataract Refract Surg (submitted. 2010).

42. Vinciguerra P, Albè E, Trazza S, et al. Refractive, topographic, tomographic, and aberrometric analysis of keratoconic eyes undergoing corneal cross-linking. Opbthalmology. 2009;116:369378 . 